Revista de la Facultad de Ingeniería Industrial

19(1): 69-77 (2016) UNMSM

ISSN: 1560-9146 (Impreso) / ISSN: 1810-9993 (Electrónico)

\title{
Análisis en la Empresa Gráfica Lao, con las métricas del modelo Scor
}

\section{RESUMEN}

En este artículo se presenta, la relevancia que tiene el modelo Scor para efectuar un análisis como se encuentra una empresa, tal como se demuestra en esta investigación y revertir las deficiencias.

Palabras clave:Modelo Scor, métricas y atributos.

Lao Graphic Enterprise, ANalysis with MODEL METRICS SCOR

\section{ABSTRACT}

In this article, the relevance of the Scor model for an analysis as is a company presents itself as demonstrated in this research and reverse the deficiencies.

Keywords: Model Scor , metrics and attributes

\section{INTRODUCCIÓN}

La gráfica Lao es una importante compañía en el rubro de confecciones de formatos continuos de dos y tres copias, su principal insumo es el papel autocopiante en sus tres modalidades que existen denominados Coated Back (CB recubierto al reverso), Coated Back and Front (CBF recubrimiento en el frente) y en el reverso y Coated Front (CF recubierto del frente); estas bobinas de papel, son importadas tanto de Estados Unidos como de China, en el Perú no se fabrican estos productos.

Sus principales clientes están definidos en dos grupos: los que son por concurso público que son las entidades que pertenecen al estado y los clientes privados.

La característica de la realización de los trabajos es a pedido, ya que cada uno cuenta con su propio logo, RUC y además de diseño en la estructura de las hojas, entre otras características.

En los últimos meses se ha venido produciendo considerables retrasos en las entregas en especial a los privados y mínimo a las entidades estatales producto de que tienen una fecha de entrega, en caso que no se cumpla hay una penalidad, generando incomodidad, malestar y sobretodo desconfianza como consecuencia de esta situación un $7 \%$ de los clientes ya no trabajan con la gráfica Lao.

En este artículo de investigación se proporcionará las métricas que da el modelo Scor, la metodología a desarrollar, asimismo les permitirá a otras empresas determinar su situación en que se encuentran y revertir dicha realidad.

\section{MARCO TEÓRICO}

\subsection{Modelo Scor}

Fue creado en 1996 por el Supply Chain Council (SCC), SCOR es un acróstico de las palabras Supply Chain Opertaions Reference, es un modelo de referencia, en el cual se detallan los procesos del giro del negocio y lo más resaltante es el cumplimiento con los requerimientos del cliente.

* Docente de la Universidad San Juan Bautista e ICAM, egresado de la maestría en Gestión de Operaciones y Servicios Logístico. E-mail: npapanicolaud@gmail.com

** Docente FIIS, egresado de la maestría en Gestión de Operaciones y Servicios Logístico. E-mail: luis.evangelista@yahoo.es 
En el resumen ejecutivo del 2010 el SCC indica que se cuenta con más de 200 procesos, 550 métricas, 500 mejores prácticas.

Está organizada por 5 principales procesos que interactúan entre sí que son la de planear, abastecer, transformar, entregar y devolver, tal como aparece en la Figura 1. En una visión micro de la cadena de suministro.

En esta figura se ve que lo principal es la planeación, las coordinaciones con los proveedores y con los clientes que requieren el servicio de la fabricación de un producto

En la Figura 2, en una visión macro, que es el modelo Scor, que va del proveedor del proveedor hasta el cliente de los clientes, en la cual se cumplen los 5 procesos.
Chevillard (2006) menciona que el modelo Scor se aplica a todo lo concerniente a los flujos de materia prima, productos terminados, equipos, repuestos, servicios entre otros, además de la recepción del pedido hasta el pago de la factura. Lo que no incluye son: generación de la demanda, innovación, tecnología y marketing.

\subsection{Procesos}

Como se indicó anteriormente existen 5 procesos que a continuación se detalla.

- Planeación. Se encarga de equilibrar la demanda, en primer lugar que no exista un desabastecimiento o rotura de stock por eso es importante calcular el tiempo del pedido, asimismo ver cuánto hay en stock. Por eso es importante la comunicación entre las áreas

Figura 1. Estructura de la cadena de suministro.

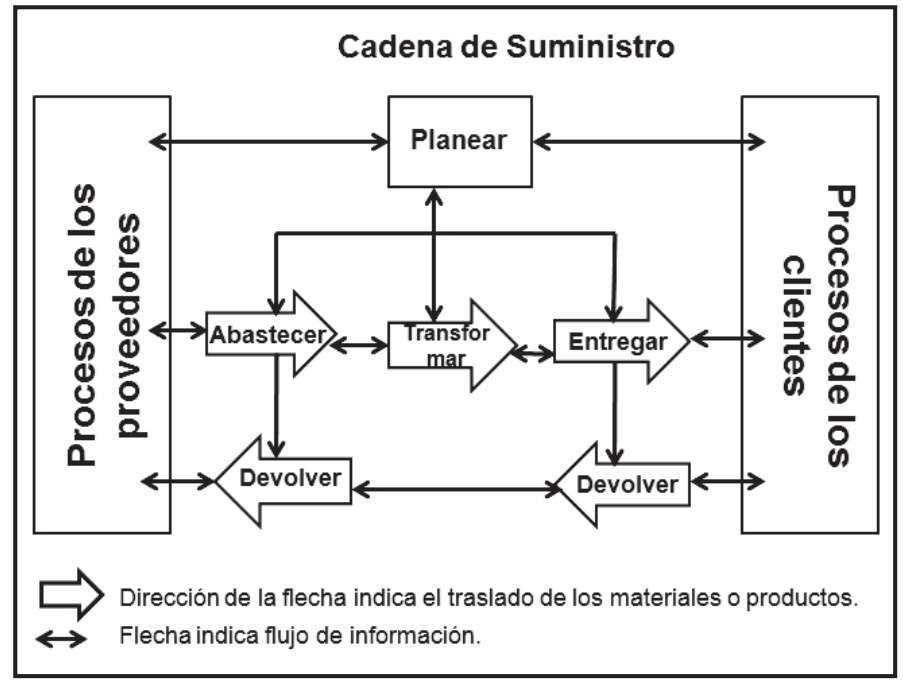

Fuente: Resumen ejecutivo (2010) SCC, elaboración propia.

Figura 2. Modelo Scor.

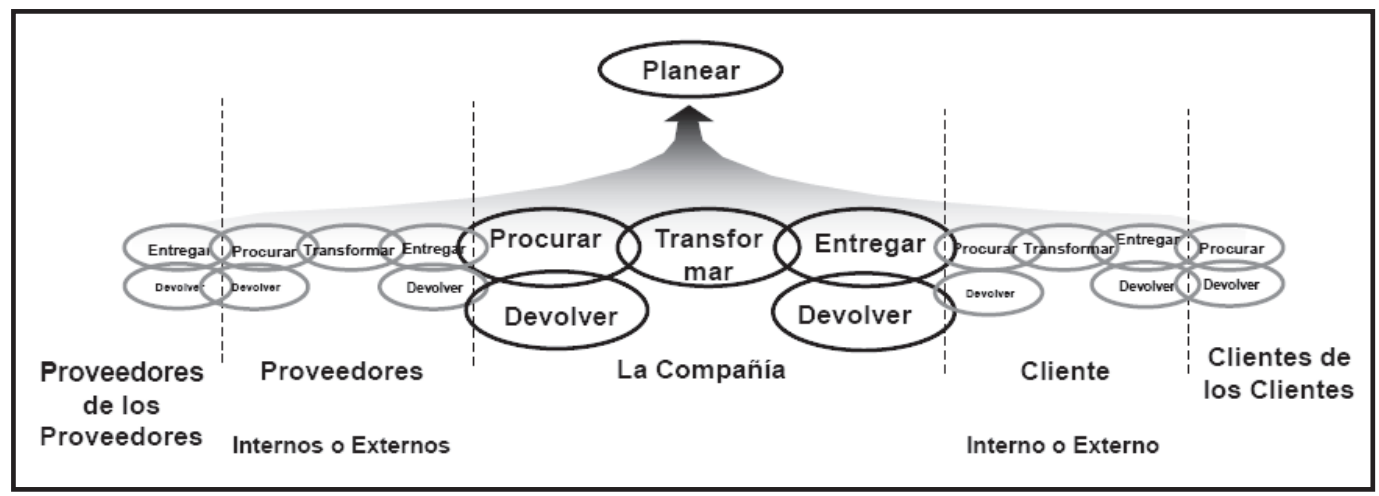

Fuente: Supply Chain Council (2010). 
involucradas. Además es la que debe planificar todos los pasos a seguir para cumplir con los tiempos y entregas establecidos.

- Abastecimiento. Responsable de los requerimientos con tiempo, contar con el espacio físico suficiente para la recepción de la mercadería, mantener en orden el almacén y llevar un control de inventario de la materia prima o productos existentes, entre otros.

- Producción. Para producir se requiere la materia prima para hacer la transformación en un producto terminado o semielaborado, añadiéndole un valor, lo principal es que dicha área solicite con tiempo los requerimientos a abastecimiento, también es la responsable que todos los equipos estén en óptimas condiciones, entre otros.

- Entrega. Al culminar el proceso de producción se coordina el envío del producto al cliente, que puede ser por ventana horaria para facilitar y ahorrar costos, en dicho proceso se verifica los ítems de la orden del pedido con sus respectivas cantidades, antes del traslado respectivo.

- Retorno. Son las devoluciones no solamente del producto o mercadería incluye los documentos recepcionados, es como consecuencia de que no es el producto requerido, llegó averiado, es otra tonalidad, generando malestar al cliente por el incumplimiento del pedido.

\subsubsection{Niveles y categorías de procesos}

Existen 4 niveles en este modelo, el último esta fuera del proyecto: que está conformado de la siguiente manera.

\section{Superior (tipos).}

2. Configuración (categorías).

3. Elementos (descomposición).

4. Implementación (elementos de la descomposición).

Tal como aparece en la Figura 3.

Con relación al nivel superior es el principal, ya que es el que va a permitir hacer la diferencia y tener ventaja competitiva con el resto de empresas.

En el de configuración, existen 26 categorías estandarizadas, que se dividen de la siguiente manera: 5 en planificación, 16 en la configuración y 6 de apoyo, en la figura 4, se detalla cada uno de ellos.

Referente al nivel de apoyo, su característica es que no tiene indicadores, solo de enlace con las demás categorías.

\subsection{Atributos y métricas}

El Banco Interamericano de Desarrollo dictó en el mes de Diciembre del 2015 un curso MOOC denominado "Datalogía: la toma de decisiones basada en datos", en la cual explica la importancia

Figura 3. Niveles del modelo.

\begin{tabular}{|ll|l|l|l|l|}
\hline & & Niveles & Descripción & Esquema & \\
\hline & & 1 & & & \\
\hline
\end{tabular}

Fuente: Pires, S., y Carretero, D. L. (2007). 
Figura 4. Procesos del modelo Scor.

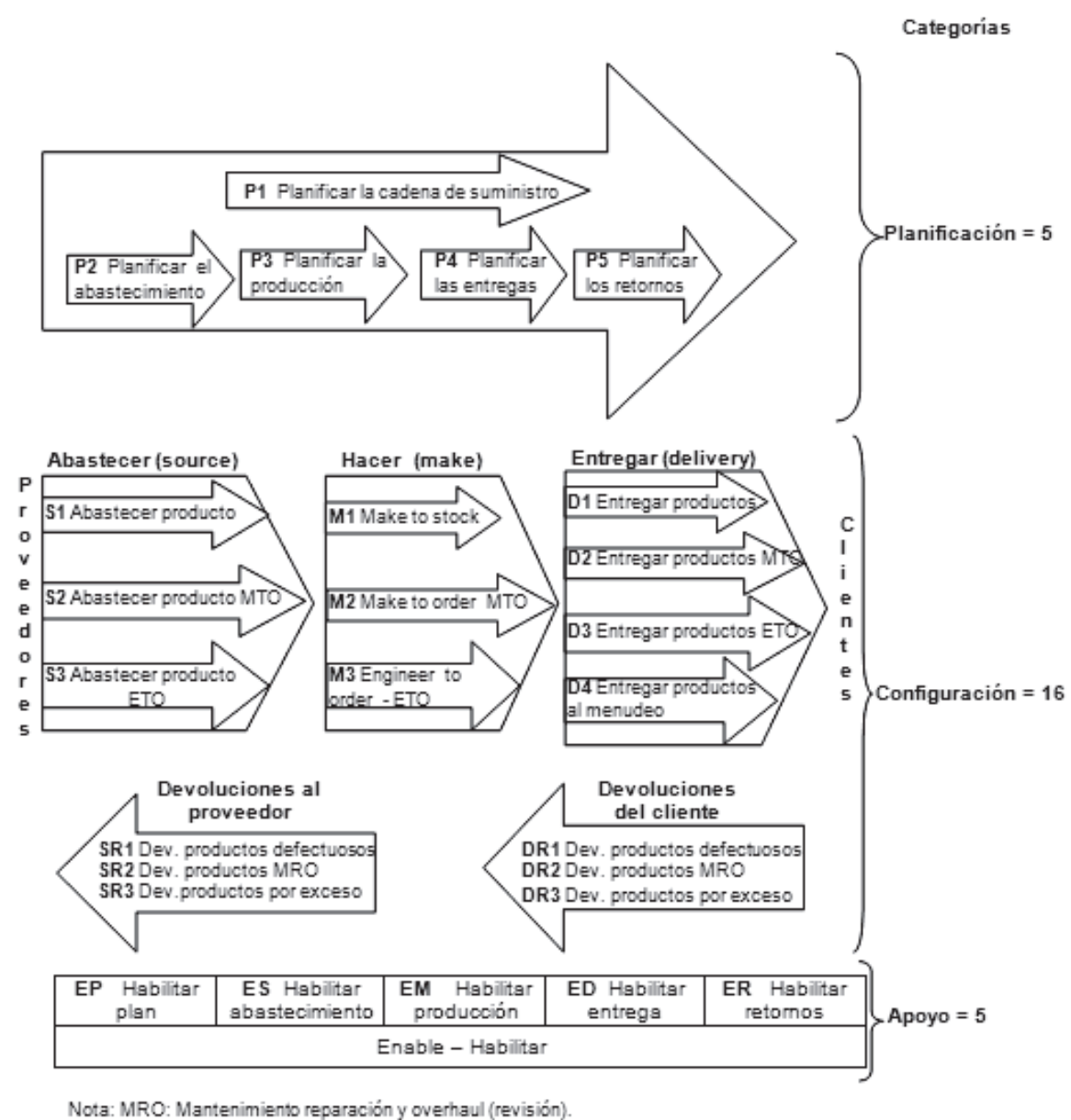

Fuente: Pires, S., y Carretero, D. L. (2007) y elaboración propia.

que tienen los indicadores para tomar medidas que puede ser de orden correctivas o de destacarse con relación a otras compañías del mismo giro.

Se ha efectuado esta pequeña introducción porque a continuación se va a explicar los indicadores estratégicos de los 5 atributos que a continuación se detallan y están en la Tabla 1.

\section{Confiabilidad}

Esto involucra no solamente la entrega en la fecha programada y en las cantidades solicitadas, incluye toda la documentación de envío y recepción, además de las condiciones de embalaje acordadas previamente.

\section{Capacidad de respuesta}

Se refiere a la rapidez que uno pueda atender al cliente, cuando hace un requerimiento, es importante contar con planes de contingencia, para satisfacer la demanda.

\section{Agilidad}

Está relacionado con el anterior, se asocia con los factores endógenos y exógenos que se puedan presentar tales como, el retiro de un accionista, huelga dentro de la empresa, incremento o disminución de la demanda, desastres naturales entre otros, la compañía tiene que tener inmediatez para afrontar la coyuntura que se dé.

\section{Costos}

Son todos los gastos en que se incurre en toda la cadena de suministro desde el transporte, manipuleo, estiba, desestiba, mano de obra, producción entre otros, es importante tener liderazgo de costos como lo menciona Porter, para contar con una ventaja competitiva.

\section{Activos}

Son los bienes tangibles que cuentan una empresa, maquinarias, almacenes, camiones, que le permitan atender los pedidos en todo el proceso. 


\section{DESCRIPCIÓN Y SITUACIÓN DE LAEMPRESA}

Como se ha mencionado anteriormente esta es una empresa dedica a la fabricación de formatos continuos de dos o tres copias, de acuerdo al modelo es M2 (make to order) hacer por pedido, como se ve en la figura $n^{\circ} 5$ del diagrama de flujo que se inicia desde la recepción de la orden de compra se verifica si ya es cliente en el área de arte, para buscar la placa respectiva, luego de los vistos buenos pasa a producción, culminada esta etapa pasa a despacho y se coordina la entrega.

La empresa gráfica cuenta con tres principales proveedores, dos de Estados Unidos y uno de China, cuando se trae las bobina de este último país se debe pagar en aduanas el derecho ad valorem de la mercadería encareciendo el producto, aparte del viaje que es más largo, en la figura $n^{\circ} 6$ se visualiza como es el flujo en macro la cadena de suministro con relación a los clientes, se hace la diferencia entre lo estatal (público) y privado, el primero lo ve el área de administración por ser licitaciones en cambio el segundo lo maneja ventas.

El punto crítico de la empresa es con los plazos pactados de entrega, los cuales no se cumplen al $100 \%$ en la fecha acordada.

Se efectuó una muestra en los 3 primeros meses del año 2015 en las gráficas siguientes se ha divido en dos grupos, por un lado están las órdenes totales de las cuales cuántas se entregaron completas e incompletas y esta última se ha dividido por clientes (entidades) públicas y privadas, ambas están en porcentajes.

En el mes de enero el total de órdenes que se debieron entregar fue de de 267 : sólo el $65 \%$, se entregaron completas en la fecha pactada (173), y un $35 \%$ se remitieron con un retraso de 1 a 4 días (94), como se ve en la figura $n^{\circ} 7$, de ese porcentaje que no se entregó a tiempo un $85 \%$ fue para los clientes privados (80) y un $15 \%$ de retraso para las empresas públicas (14), Figura 9.

Tabla 1. Atributos y métricas

\begin{tabular}{|c|c|c|}
\hline & Atributos & Métricas estratégicas \\
\hline \multirow{3}{*}{ 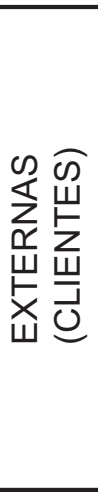 } & Confiabilidad & $\begin{array}{l}\text { Cumplimiento de la orden perfecta. } \\
\text { (Total de órdenes perfectas) / (Total de órdenes) }\end{array}$ \\
\hline & $\begin{array}{l}\text { Capacidad de } \\
\text { respuesta }\end{array}$ & $\begin{array}{l}\text { Tiempo de ciclo de cuplimiento de la orden. } \\
\text { (Sumatoria de todos los tempos de ciclo de las órdenes } \\
\text { enviadas) / (Total de órdenes enviadas) }\end{array}$ \\
\hline & Agilidad & $\begin{array}{l}\text { Flexibilidad de la cadena de suministro. } \\
\text { El mayor del núcleo de días requeridos para lograr un } \\
\text { incremento permanente en abastecimiento,producción y } \\
\text { entrega. } \\
\text { Adaptabilidad de la cadena de suministro. } \\
\text { Aguas arriba; Porcentaje de incremento permanente. } \\
\text { Aguas abajo: Porcentaje de reducción permanente. }\end{array}$ \\
\hline \multirow{4}{*}{ 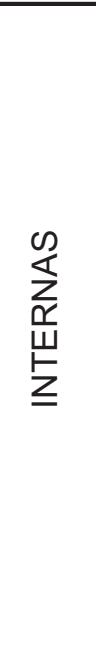 } & Costos & $\begin{array}{l}\text { Costo de la gestión de la cadena de suministro. } \\
\text { (costo de plan) + (costo de abastecer) + (costo de } \\
\text { entregar) }+ \text { (costo de devolver).. } \\
\text { Costo de los bienes vendidos } \\
\text { Materiales directos + MOD + gastos generales. }\end{array}$ \\
\hline & \multirow{3}{*}{ Activos } & $\begin{array}{l}\text { Tiempo de ciclo de efectivo a efectivo. } \\
\text { (Días de inventario con suministrs) + (días de cuentas a } \\
\text { cobrar) - (días de cuentas a pagar). }\end{array}$ \\
\hline & & $\begin{array}{l}\text { Retorno sobre los activos fijos. } \\
\text { ((Ingresos a la cadena de suministro) - (costos de venta) } \\
\text { - (Costo de la gestión de la cadena de suministro)) / } \\
\text { (activos fijos de la cadena de suministro). }\end{array}$ \\
\hline & & $\begin{array}{l}\text { Retorno sobre el capital de trabajo. } \\
\text { ((Ingreso de la cadena de suministro) - (costos de ventas) } \\
\text { - (costo de la gestión de la cadena de suministro)) / (capital } \\
\text { de trabajo). }\end{array}$ \\
\hline
\end{tabular}

Fuente: Render B y Heizer J. (2014), elaboración propia. 
Figura 5. Diagrama de flujo.

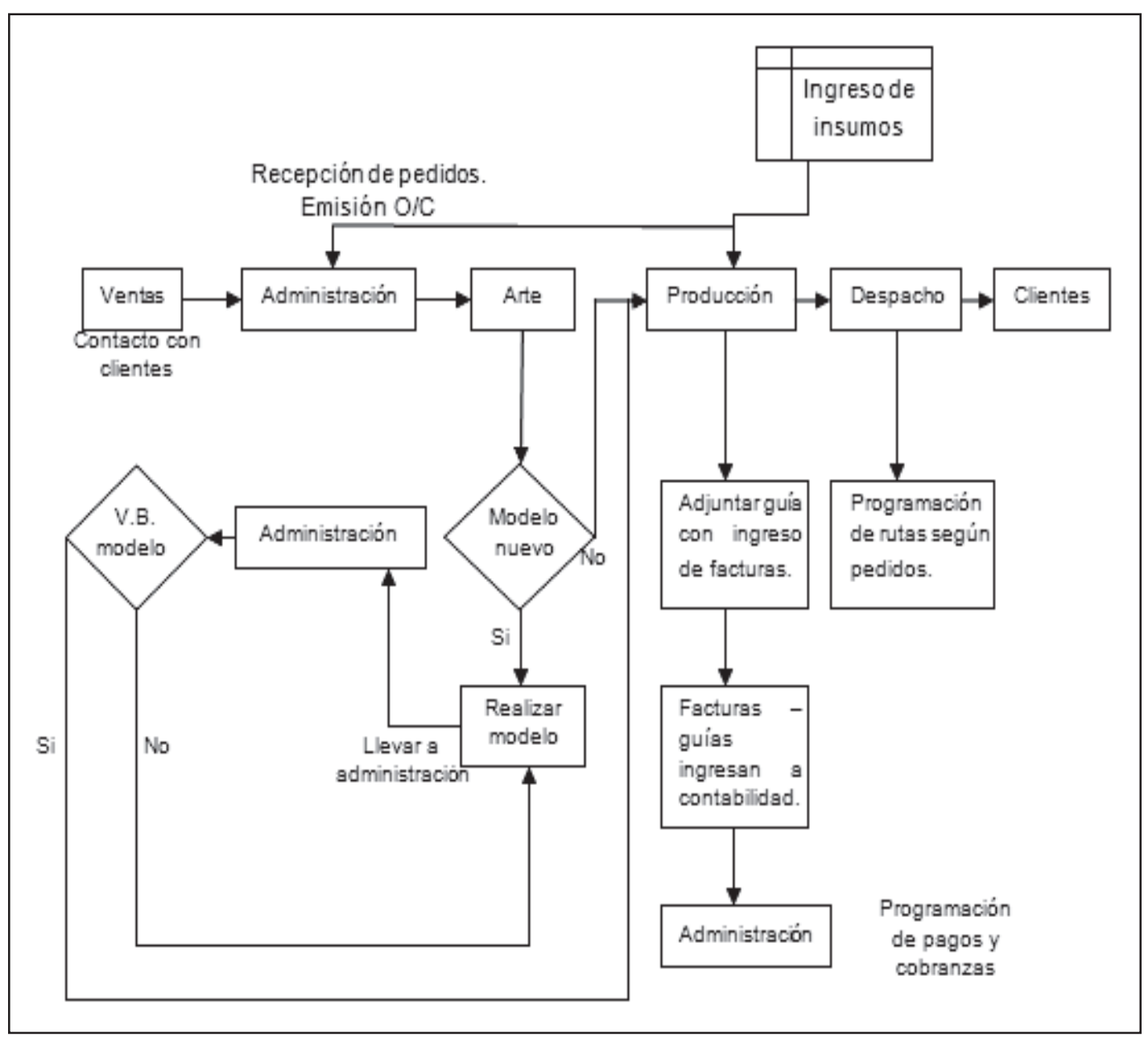

Fuente: Empresa gráfica Lao.

Figura 6. Diagrama de proveedores - empresa clientes.

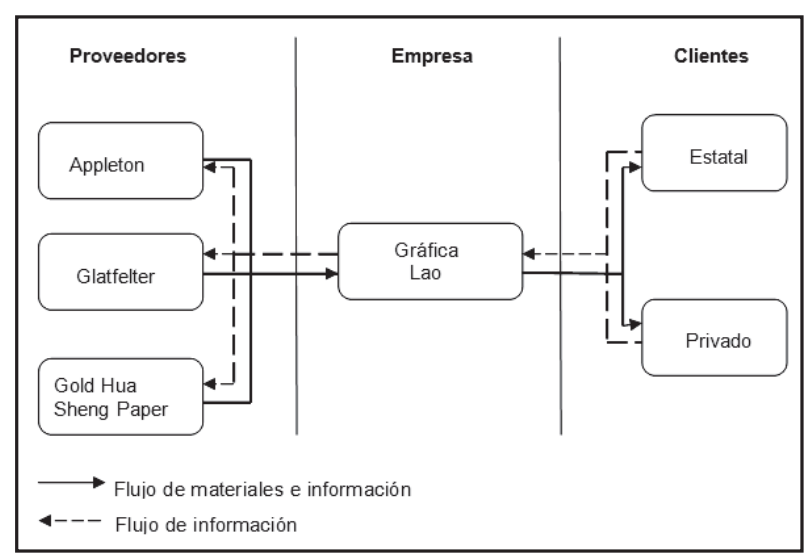

Fuente: Elaboración propia. 
Figura 8. Órdenes Enero.

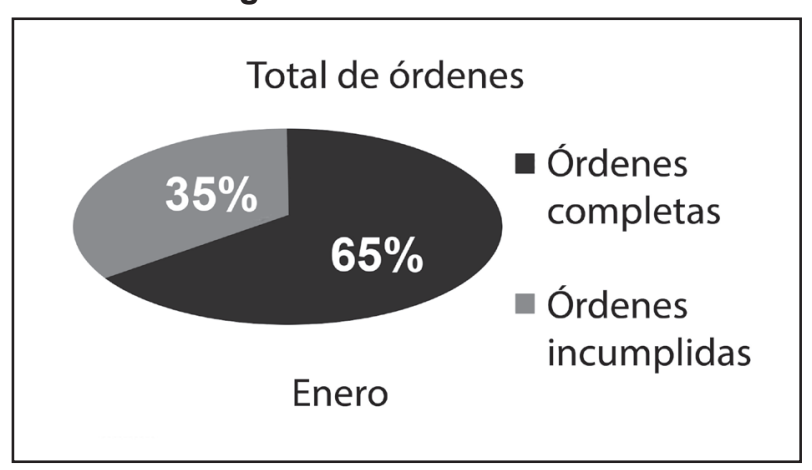

Fuente: Elaboración propia.

Febrero no fue ajeno a la situación de incumplimiento del total de órdenes 200 , solo el $60 \%$ se cumplió su entrega en la fecha establecida (120) y un $40 \%$ de órdenes incumplidas (80) figura $n^{\circ} 10$, de este número un $91 \%$ fue a las entidades privadas (73) y un $9 \%$ a las entidades públicas (7), Figura 11.

En el último mes de estudio Marzo, reinciden en los retrasos de un total de 131 órdenes, un $66 \%$ de cumplimiento (87) y un $34 \%$ de no entrega en la fecha acordada (44) Figura 12, de este porcentaje

Figura 10. Órdenes Febrero.

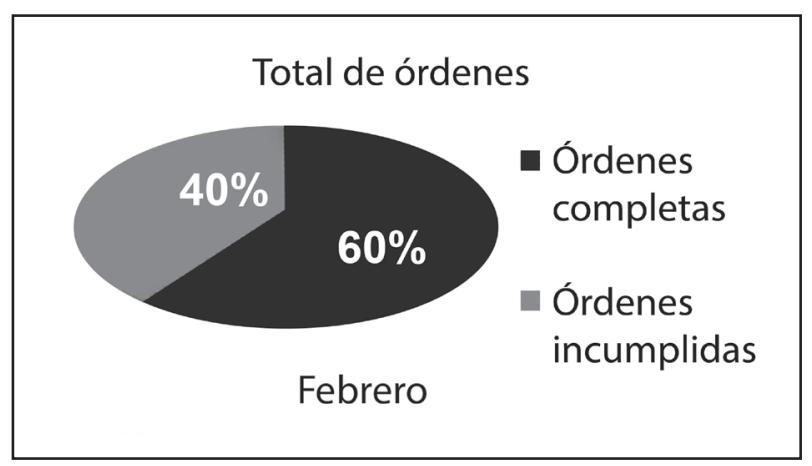

Fuente: Elaboración propia.

Figura 12. Órdenes Marzo.

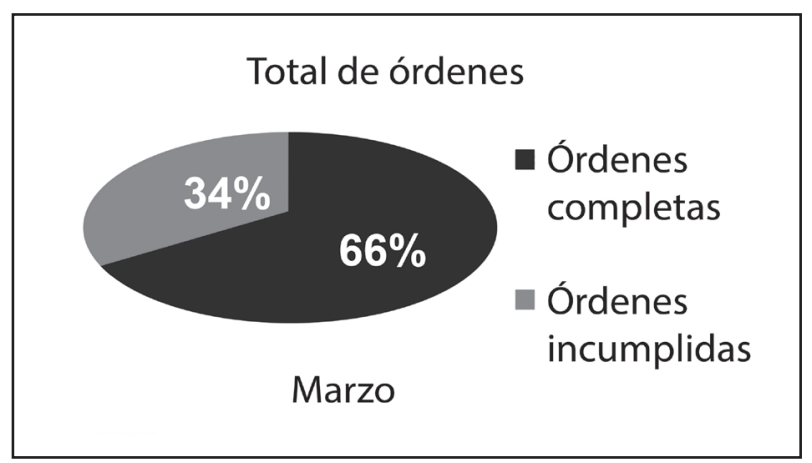

Fuente: Elaboración propia.
Figura 9. Incumplimiento Enero.

Incumplimiento de entrega a las entidades

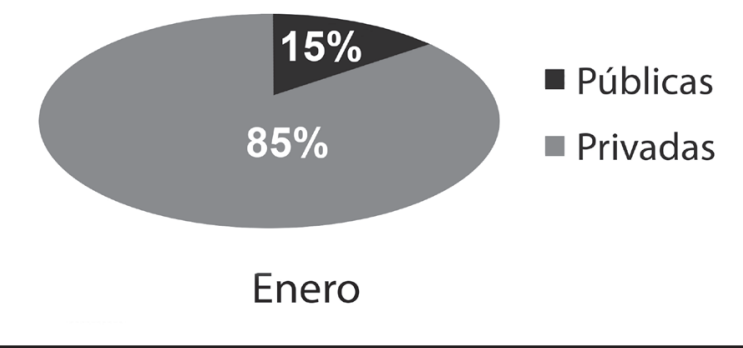

Fuente: Elaboración propia.

último un $89 \%$ es para las entidades privadas (39) y $11 \%$ para las públicas (5), ver Figura 13.

Como se aprecia el rango de entregas completas en la fecha establecida, está entre un $60 \%$ a $66 \%$ es un indicador que no corresponde a los estándares de confiabilidad, referente a que clientes se incumple es a las entidades privada en promedio es un $88 \%$ y un $12 \%$ a las públicas, las entregas de estos retrasos se da en un periodo de 1 a 5 días posterior a la fecha pactada.

Figura 11. Incumplimiento Febrero.

Incumplimiento de entrega a las entidades

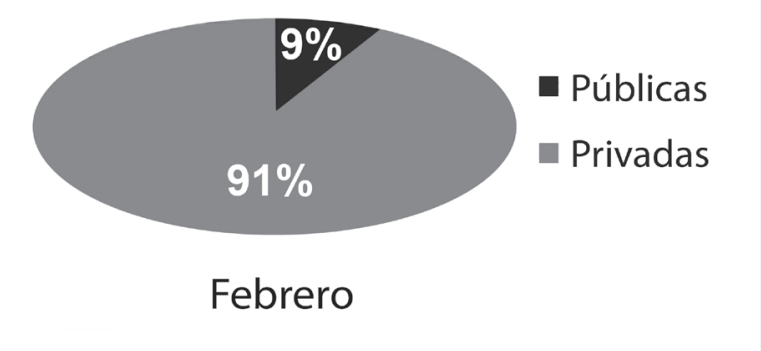

Fuente: Elaboración propia.

Figura 13. Incumplimiento Marzo.

Incumplimiento de entrega a las entidades

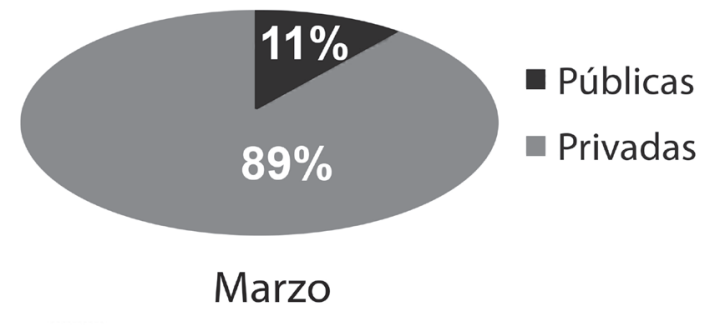

Fuente: Elaboración propia. 


\section{METODOLOGÍA}

Dado este escenario, para determinar cuáles fueron las causas que han llevado a este punto crítico de la empresa gráfica, se utilizó la matriz de Vester ver Tabla 2 y para determinar los componentes, se efectuó un análisis interno y se fijó 4 áreas de la empresa y 1 referente a la especialización de los técnicos, que eran las que tenían mayor incidencia con relación al resto de otras áreas.

Trasladando los resultados de los matriz a la figura 14 donde se divide en 4 cuadrantes, se observa que:

En el cuadrante de críticos, de los 5 componentes de la matriz hay 3 , lo cual es alarmante y preocupante y que son las causas en los retrasos en las entregas y son: Administración, producción y ventas, como consecuencia de la división de clientes, generando caos y presiones a producción para que hago primero su orden. Especialización de técnicos está en el medio de los 4 cuadrantes, por el mismo desorden que se ha generado y está en una situación que se puede agravar si no se toman los correctivos necesarios y despacho está en la línea media de indiferentes con pasivos, en ellos no hay problema ni bien tengan el pedido lo remiten al cliente.

\section{CONCLUSIONES}

Con el modelo Scor, con sus atributos y métricas se ha podido detectar los problemas que tiene la empresa gráfica.

Asimismo con el modelo se puede adaptar las buenas prácticas de otras empresas del rubro a la empresa gráfica.

Tabla 2. Matriz de Vester

\begin{tabular}{|c|l|c|c|c|c|c|c|}
\cline { 2 - 9 } \multicolumn{1}{c|}{} & \multicolumn{1}{c|}{ Decripción } & A & B & C & D & E & Activos \\
\hline A & Administración & & 3 & 2 & 2 & 2 & 9 \\
\hline B & Ventas & 3 & & 2 & 1 & 1 & 7 \\
\hline C & Producción & 3 & 1 & & 2 & 1 & 7 \\
\hline D & Especialización de técnicos & 2 & 0 & 3 & & 0 & 5 \\
\hline E & Despacho & 2 & 2 & 1 & 0 & & 5 \\
\hline & Pasivos & 10 & 6 & 8 & 5 & 4 & \\
\hline
\end{tabular}

Fuente: Elaboración propia.

Figura 14. Detalle de problemas.

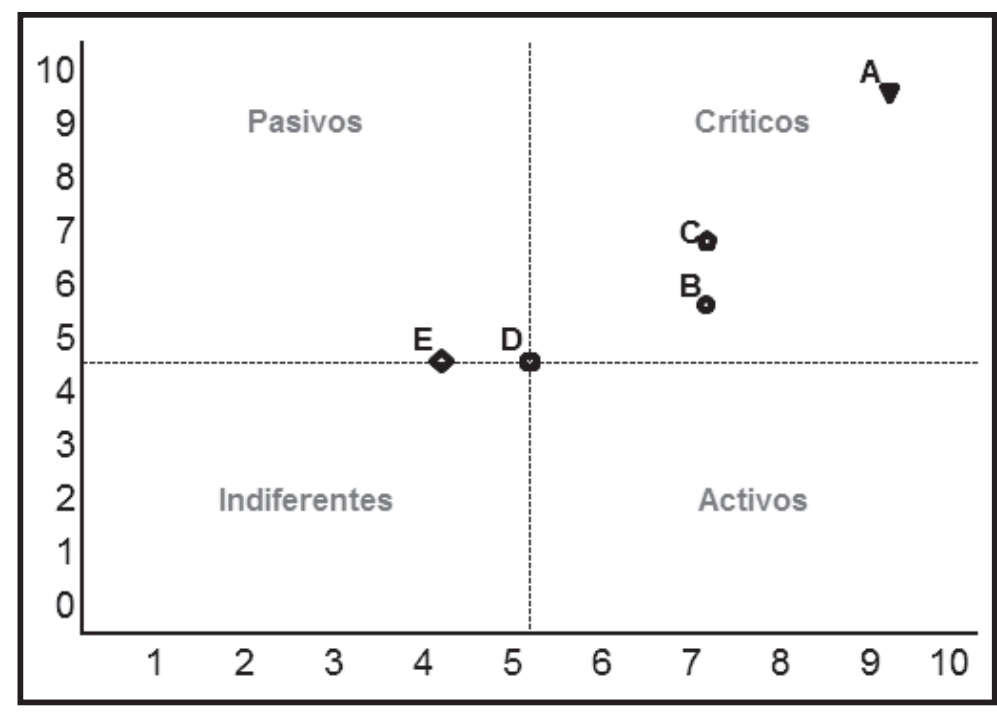

Fuente: Elaboración propia. 
Con el modelo se puede organizar toda la cadena de suministro, de acuerdo a los niveles y la configuración con sus respectivos enlaces.

Se debe tomar una decisión con respecto a quién va a ver las ventas en forma general e implementar nuevas políticas.

\section{REFERENCIAS BIBLIOGRÁFICAS}

[1] Appleton papers. (1997). Manual soluciones técnicas. Papel autocopiante. Estados Unidos. Appleton papers. (2012, 4 de agosto).

[2] Banco Interamericano de Desarrollo: Datalogía: La toma de decisiones basadas en $\mathrm{d}$ a $\mathrm{t}$ o $\mathrm{s}$. Curso MOOC. Disponible en https://courses.edx. org/courses/course- v1:IDBx+IDB10x+2015 T4/courseware/c6 b5 11 eeb $74444939 \overline{7}$ ef6f4b455e7968/ (2015, 20 de diciembre).

[3] Campos; J, Cruz, C y Sánchez, J. (2012). Diagnóstico basado en el Modelo Scor para I a cadena de suministro de la empresa Matecsa S.A. Disponible en http://www.unilibre.edu.co/ revistaavances/avances\%20_9-1/r9-1_art12. pdf. (2015, 15 de diciembre).

[4] Fontalvo, H. T. J., y Cardona, R. D. (2011). La cadena de suministro: un enfoque práctico para el diseño e implementación del modelo SCOR. Bogotá: Corporación para la gestión del conocimiento ASD 2000.

[5] Pacheco, F. (s/f). Modelo Scor. Referencias de las operaciones de la cadena de abastecimiento.
Disponible en https://consorciocalacor. wikispaces.com/.../presentación- SCOR.pptx. (2013, 20 de marzo).

[6] Pires, S., \& Carretero, D. L. (2007). Gestión de la cadena de suministros. España: McGraw-Hill España.

[7 Poluha, R. (2007). Application of the SCOR model in Supply Chain Management. New York: Cambrian Press.

[8] Render, B., y Heizer, J. (2014). Administración de Operaciones. (9a. ed). México DF: Pearson Educación.

[9] Ribate, J. (2011). Modelo de referencia SCOR. Disponible en http://www.aragonempresa. com/descargar.php? $a=50 \& t=$ paginas_we $b \& i=131 \& f=$ ea $1 b f 8 b f d 81$ cfe 6 b254f2a 324 effadb2. (2014, 6 de julio).

[10] Schroeder, R., Goldstein, S. M., y Rungtusa natham M. J. (2011) Administración de operaciones. Conceptos y casos contemporáneos (5.a ed). México D.F.: McGraw-Hill Interamericana.

[11] Supply Chain Council (2009). El modelo Scor. Resumen ejecutivo. Disponible en http://www.apics.org/sites/apics -supply-chain-council. (2012, 11 julio). $\quad[p h p ? a=50 \& t=p a g i n a b \& i=131$ \& $f=\begin{array}{llllll}e & a & 1 & b & f & 8\end{array}$ b f d 81 c fe 6 b 254 f 2 a 324 e ffa d b 2 . (2014, 6 de julio). 